\title{
El Eterno Retorno. ¿Son fascistas las ideas-fuerza de la Nueva Derecha Europea (ND)?
}

\author{
Joan ANTÓN-MELLÓN
}

Recibido: 25 de mayo de 2011.

Aceptado: 2 de agosto de 2011.

\section{RESUMEN}

El artículo analiza las concepciones nucleares o ideas-fuerza de la Nueva Derecha Europea (ND) en sus diferentes versiones, ortodoxa (Alain de Benoist) y heterodoxa (Guillaume Faye, Pierre Vial), y las compara con las concepciones nucleares del fascismo clásico (1919-1945) para establecer los factores de continuidad y de divergencia con él. Los ideólogos de la ND difieren del fascismo clásico en diversos aspectos, como por ejemplo en el apoyo a las formaciones populistas de derecha radical, pero comparten una misma visión del mundo y una forma de estar en él. En sus publicaciones muestran, de forma similar a como lo hicieron los ideólogos del fascismo clásico, una misma diagnosis de la situación (decadencia); plantean unos mismos objetivos (palingénesis de la comunidad) y utilizan unos mismos referentes culturales (destacadamente a Friedrich Nietzsche y los Revolucionarios Conservadores del primer tercio del siglo veinte). Un aspecto central de los factores de continuidad reside, a su vez, en que comparten una similar concepción del hombre, de la naturaleza y de la historia. El hombre como un ser agresivo, jerarquizado y territorializado.

\section{PALABRAS CLAVE}

Nueva Derecha Europea (ND), fascismo clásico, Alain de Benoist, Guillaume Faye, Derecha Radical.

\begin{abstract}
This article analyses the elements of continuity and divergence between the core concepts of Classical Fascism (1919-1945) and those of the European New Right, in both its forms: the orthodox, embodied by Alain de Benoist, and the heterodox, represented by Guillaume Faye and Pierre Vial. Though these authors differ in terms of whether they provide explicit support for neo-populist political formations, they share a Heideggerian/Dasein vision of the world. In their publications they present the same diagnosis of the situation (spiritual and/or racial decadence), set the same priority objec-
\end{abstract}


tives (palingenesis) and use the same cultural reference points (for example, Friedrich Nietzsche and the German Revolutionary Conservatives of the first third of the twentieth century). In view of these traits, it seems justifiable to label them as twenty-first century neo-Nazis. Above all if we show (as we aim to do in this article) that their conceptions of man and nature (for example, man as an aggressive, hierarchical and territorial being) are the same as those of the ideologues of Classical Fascism.

\section{KEY WORDS}

European New Right, classical fascism, Alain de Benoist, Guillaume Faye, the Radical Right.

\section{INTRODUCCIÓN}

El presente artículo pretende, como objetivo principal, explicitar las ideas-fuerza o concepciones nucleares ${ }^{1}$ de la Nueva Derecha Europea (ND) ${ }^{2}$. A la vez, al analizar la coherencia interna de dichas premisas básicas, se aportarán elementos de juicio al debate académico sobre la adscripción ideológica de la $\mathrm{ND}^{3}$. Nuestra hipótesis principal mantendrá que dicha adscripción debe situarse, inequívocamente, en el terreno de la Extrema Derecha y/o Derecha Radical, debiéndose des-

${ }^{1}$ Martin Seliguer las denomina núcleo fundamental de una ideología, separado del operativo; en: "Fundamental and Operative Ideology: The Two Principal Dimensions of Political Argumentation": Policy Sciences, vol. 1 (1970); y Michael FrEEDEN las llama núcleo ineliminable, contrapuesto a los adyacentes o periféricos, en: "Political Concepts and Ideological Morphology": The Journal of Political Philosophy, vol. 2, n. 2 (1994).

${ }^{2}$ Tanto en su versión ortodoxa (Alain de Benoist, Charles Champetier), como heterodoxa (Gillaume Faye, Pierre Vial o Robert Steuckers). El interés de su estudio radica en que comprender las concepciones nucleares de la ND es entender la armazón ideológica de la Extrema Derecha/Derecha Radical desde el siglo veinte hasta el presente en sus principios axiológicos y ontológicos.

${ }^{3}$ Ver Thomas SheEnAn, "Myth and Violence: The Fascism of Julius Evola and Alain de Benoist": Social Research, vol. 48, n. ${ }^{\circ} 1$ (Spring 1981); Pierre-André TAguiefF, Sur la Nouvelle droite, Descartes, Paris, 1994; Roger GrifFIN, "Plus ça change! El pedigrí fascista de la Nueva Derecha”, en Miguel Ángel Simón (ed.), La Extrema Derecha en Europa desde 1945 a nuestros días, Tecnos, Madrid, 2007; Joan ANTÓN-Mellón, "La cultura e ideología política del Neopopulismo en Europa Occidental: MNR/FN (Francia), FPÖ (Austria) y Lega Nord (Italia)", en SIMÓN (ed.), La Extrema Derecha en Europa desde 1945 a nuestros dias; Tamir BAR-ON, Where have all the fascist gone?, Ashgate, England/USA, 2007; y Diego Luís SANROMÁN, La Nueva derecha, CIS, Madrid, 2008. 
echar, por tendencioso y falso, su propio análisis de que han logrado establecer un nuevo paradigma teórico-político más allá de la derecha y la izquierda. Dicha adscripción se refrendará al explicitarse las analogías y divergencias entre las concepciones nucleares de la ND y aquellas del Fascismo Clásico (1919-1945), una de las variantes de mayor relevancia de la Derecha Radical europea del primer tercio del siglo veinte.

La metodología utilizada ha consistido en clarificar y exponer la lógica nodal de los textos estudiados de la ND en torno a los siguientes parámetros clasificatorios: cómo se definen y describen (autodefiniciones); cómo analizan la situación histórica (diagnóstico); cuáles son sus aspiraciones (utopía); qué es todo aquello a lo que se oponen (objetivos); y, finalmente, cuáles sus principios ontológicos y axiológicos (visión del mundo y concepción del hombre, de la naturaleza y de la historia). Dicha coherencia interna será contrastada primero con el modelo teórico de Alain Bihr según el cual los tres factores decisivos de los idearios de Extrema Derecha en Occidente son: i) propugnar planteamientos ultra-etnonacionalistas elevando la identidad colectiva a la categoría de fetiche; ii) establecer la desigualdad como una categoría ontológica y axiológica fundamental; y iii) defender una concepción belicista de la existencia en la que la lucha está glorificada como un factor supremo de la vida ${ }^{4}$. En segundo lugar las concepciones nucleares de la ND serán cotejadas con aquellos factores constitutivos y definitorios del fascismo que diferentes científicos sociales han establecido como síntesis de sus investigaciones. Intentando así establecer las semejanzas en las diferencias halladas y, a la vez, las diferencias en las semejanzas.

Respecto a las fuentes consultadas se ha analizado en primer lugar una selección de la extensa obra del líder intelectual de la ND ortodoxa, Alain de Benoist, y de la ND heterodoxa, Guillaume Faye. En segundo lugar, se han revisado los editoriales y artículos más relevantes de las revistas Élements (primer número septiembre/octubre de 1973, órgano oficial del Groupement de Recherche et d'Études pour la Civilisation Européenne - GRECE - de la ND francesa); Trasgressioni (primer número mayo/agosto de 1986, revista teórica de la ND italiana) y Hespérides (1993-2000, revista teórica de la ND española). Y, finalmente, se han estudiado los manifiestos más relevantes. Es el caso de Manifies-

${ }^{4}$ Alain BIHR, L'actualité d'un archaïsme, Editions Page deux, Lausanne, 1999. Podemos comprobar la operatividad de dicho modelo al estudiar, por ejemplo, las concepciones nucleares del Fascismo Clásico; ver Joan AnTón-Mellón, "Las concepciones nucleares, axiomas e ideasfuerza del Fascismo Clásico (1919-1945)": Revista de Estudios Políticos, n. ${ }^{\circ} 146$ (octubre-diciembre 2009). 
to: La Nueva Derecha del año 2000, redactado por Alain de Benoist y por el actual director de Éléments, Charles Champetier, así como, desde la ND española, del Manifiesto del Proyecto Cultural Aurora; ¿Qué hacer? Elementos para un discurso de contestación, documentos aparecidos a finales del siglo veinte. También se ha analizado El Manifiesto contra la muerte del espiritu y de la tierra, aparecido en España en 2002.

Por lo que se refiere al origen y evolución de la ND europea constatamos que se trata de un conjunto de ideólogos, publicaciones y asociaciones culturales que, desde finales de los años sesenta del pasado siglo, pretenden renovar los clásicos discursos de la Derecha Radical europea de la primera mitad del siglo veinte (abandonando ineficaces nostalgias) para influir ideológicamente en todas las subfamilias de la Derecha (extrema, moderada, populista o neotradicionalista). En una época de hegemonía de los valores democráticos en Occidente, tras 1945, sus adversarios son poderosos: el cristianismo, además del pensamiento político de la Ilustración y sus correlatos de la cultura de la Modernidad. Sin embargo, la decadencia de la Izquierda Radical ha creado un vacío político-cultural que la ND quiere llenar planteando sus alternativas ideológicas a los desgastados planteamientos tecnócratas del liberalismo conservador y de la socialdemocracia. Se trata de explicitar las disfunciones de la postmodernidad (anomia, hiperindividualismo, ultramaterialismo, problemas identitarios) y ofrecer sus alternativas "capaces" de eliminar la "alienación", superar la "decadencia" y recuperar la auténtica "identidad europea".

El faro teórico, con importantes matices alternativos tácticos en los autores disidentes de la ND europea (Faye, Robert Steuckers, Pierre Vial) es la asociación cultural francesa Groupement de Recherche et Études pour la Civilisation Européenne (GRECE) fundada en enero de 1968 en Niza, y paralelamente en Paris y Toulouse, por cuarenta personajes, provenientes todos ellos de la Extrema Derecha y/o Derecha Radical francesa ${ }^{6}$. Su influencia se extiende sobre todo en las bases sociales cultas de la Derecha Radical europea, ávidas de salir del desconcierto producido por las derrotas del Fascismo Clásico (1909-1945) y de los gobiernos colaboracionistas de la Europa ocupada, así como por la posterior deslegitimación de los idearios de la Derecha Radical por el peso que el antifascismo ha tenido, política y culturalmente, en la segunda mitad del siglo veinte. La influencia de GRECE cristaliza en la creación de asociaciones o grupos en

\footnotetext{
${ }^{5}$ Marco Revelli, "La nuova destra": Iride, n. 18 (mayo-agosto 1996).

${ }^{6}$ Ver Taguieff, Sur la Nouvelle droite; Ariane Chevel d'Appollonia, L'Extrême droite en France, Complexe, Bruxelles, 1996.
} 
diferentes países europeos con una mayor o menor relevancia, los más destacables en Italia, Bélgica, Gran Bretaña, España y Rusia. La revista francesa Éléments, por ejemplo, inspira la italiana Elementi, la rusa Elementy, la belga (Flamenca) Elemente y la alemana Elemente, la española Hespérides o la británica The Scorpion.

La cúspide de su influencia cultural en Francia la logran en la década de los ochenta $^{7}$ disminuyendo gradualmente en fechas posteriores. En su trayectoria, el resto de asociaciones europeas no han logrado rebasar los límites de la base social de Derecha Radical y de los nostálgicos de Extrema Derecha, aunque en su haber debe constatarse el importante logro de haber apoyado, culturalmente, los planteamientos ideológico-doctrinales de los Partidos Populistas europeos de Derecha Radical tipo FN/MNR (Francia); FPÖ/Alianza por el Futuro (Austria) o PxC (Cataluña-España).

El radical mantenimiento de una opción metapolítica (y el desprecio por la política) ha conducido a GRECE a periódicas rupturas de relevantes personajes, la mayoría de los cuales han recalado en formaciones política populistas de Derecha Radical (FN o el MNR) e incluso en partidos liberal-conservadores. Es el caso del profesor medievalista Pierre Vial (fundador de GRECE), que se sumó al FN y con posterioridad apoyó la escisión, en 1996, del MNR de Bruno Mégret (también fundador de GRECE) creando, en ese mismo año, una asociación cultural espiritualista neonazi: Terre et Peuple ${ }^{8}$.

Otro disidente destacado es Faye, la voz de mayor importancia intelectual de GRECE juntamente con el propio Benoist hasta su abandono en 1986. Típico producto del Mayo del 68 derechista, al retornar a la palestra intelectual, a finales del siglo veinte, analiza las causas de la pérdida de influencia de GRECE, critica a fondo la opción metapolítica por inoperante y reafirma los criterios básicos de la ND desechando los eufemismos y alambicaciones enmascaradoras, políticamente correctas, tan profusamente utilizadas por la ND ortodoxa. Él quiere, como Friedrich Nietzsche (1844-1900), la transmutación de todos los valores hegemónicos desde la Ilustración y un mundo heterogéneo formado por grandes unidades territoriales. Homogéneas étnicamente y con unas radicales desigualdades estructurales sociales y productivas para asegurar la sostenibilidad ecológica del sistema y el progreso de los pueblos?

${ }^{7}$ Ver: Michalina Vaughan, "Nouvelle droite: Cultural Power and Political Influence", en David S. Bell (ed.), Contemporary French Politics, Groom Helm, London/Camberra, 1982.

${ }^{8}$ Ver: Christopher Flood, "The Cultural Struggle of the Extreme Right and the Case of Terre et Peuple": Contemporary French Civilization, vol. 24, n. ${ }^{\circ} 2$ (2000).

${ }^{9}$ Guillaume FAYE, Archéofuturisme, L'Aencre, Paris, 1998. 


\section{Autodefiniciones}

La ND europea, a lo largo de su recorrido histórico, se ve a sí misma como una variante radical y especial de la derecha y como los conservadores revolucionarios de su época. En 1994 el propio Benoist afirmaba que la ND era un disidente de la derecha institucionalizada ${ }^{10}$. Por su parte José Javier Esparza, una de las figuras históricas más relevantes de la ND española, expuso que se habían cubierto varias etapas que podían describirse como un continuo alejamiento de la derecha convenciona ${ }^{11}$. Dada su opción táctica de actuación metapolítica, la ND europea se define en sus textos más emblemáticos como un "laboratorio de ideas", una "escuela de pensamiento", una "comunidad de espíritu" y también como un "espacio de resistencia contra el sistema".

Esa opción metapolítica, rechazada por la ND heterodoxa, se apoya en Antonio Gramsci (1891-1937) y su concepto de hegemonía, en una lectura del pensador comunista italiano que Faye califica, en sus críticas de finales del siglo pasado, de superficial. Se trata de que la conquista del poder político debe estar precedida del triunfo - la hegemonía - en el combate ideológico-cultural. Evidenciándose con ello su gran pragmatismo y eclecticismo teórico. Utilizan todo aquello que pueda ser útil para la defensa de sus concepciones nucleares ya provenga de la derecha como de la izquierda: Nietzsche, Martin Heidegger (18891976), Julius Evola (1898-1974), Gramsci, Carl Schmitt (1888-1985), Konrad Lorenz (1903-1989), Louis Dumont (1911-1998), Arthur Koestler (1905-1983), Giorgio Locchi (1923-1992), Georges Dumezil (1898-1986), Louis Rougier (1889-1982) o los conservadores revolucionarios del primer tercio del siglo veinte. Todo puede valer para la causa, en una exhaustiva labor de búsqueda de argumentos de autoridad intelectual, obviamente, desde una dimensión política y no científica, con grandes dosis de disonancia cognitiva cultural.

La ND europea pretende desarrollar una triple labor de desvelar las causas profundas de la decadencia de la civilización europea; realizar una acción de ingeniería ideológico-cultural para influir sobre la opinión pública; y, en tercer lugar, liderar intelectualmente a la sociedad en la superación de la decadencia y en la edificación de una comunidad política europea armónica y poderosa que, autorreconociendo su pasado identitario auténtico, renazca y construya su futuro en el presente. De ahí que su combate sea cultural pero también político. Sus

${ }^{10}$ Alain DE BENOIST, Le grain de sable. Jalons pour une fin de siècle, Le Labyrinthe, Marsat, 1994, p. 15.

${ }^{11}$ José Javier EsparZA, "La Nueva Derecha en su contexto": Hespérides, n. ${ }^{\circ}$ 16/17. 
oponentes/enemigos son muy poderosos y por ello ha habido que empezar por lo más elemental, en sus propias palabras, crear "un espacio de resistencia contra el sistema" ${ }^{12}$. Este espacio debe gradualmente ampliarse hasta lograr vencer ideológica y políticamente a las nefastas, en su opinión, consecuencias políticas y socioculturales del despliegue de la Ilustración y la posterior modernidad ${ }^{13}$. Aunque, según su óptica analítica, el problema principal es anterior: el triunfo del cristianismo.

Esta es la razón por la que una importante definición, sintética y global, del año 2000 de la ND ortodoxa defina a ésta como: "comunitaria, ciudadana, europea y pagana" ${ }^{14}$. Su lectura enfatiza que los ideales de la Ilustración son una mera laicización del cristianismo y que este ha sido un nefasto cuerpo extraño, totalmente ajeno a las raíces indoeuropeas de nuestra cultura. A su vez, el liberalismo y el socialismo son analizados como epifenómenos muy negativos de la Ilustración y el cristianismo.

La ND es un laboratorio de ideas que, según sus propios análisis, ejerce una imprescindible labor de ingeniería cultural en un adocenado mundo burgués occidental liderado por Estados Unidos, que ha transmutado su judeo-cristianismo en la hegemónica doctrina de los Derechos Humanos (el mínimo común denominador de las doctrinas igualitarias). En realidad, en su opinión, una moral del rebaño — en la terminología de Nietzsche — destinada a alienar a unas masas de población occidentales embrutecidas por el alienante consumismo e infantilizadas por el Estado-Providencia ${ }^{15}$. De esta forma la ND se ve a sí misma como "una promesa de renovación en el corazón del invierno gris y frío...una aventura del espíritu" 16 de "pesimistas activos" mientras dure el interregno ${ }^{17}$. La época

\footnotetext{
${ }^{12}$ Manifiesto de la ND española: ¿Qué hacer? Elementos para un discurso de la contestación.

${ }^{13}$ En palabras de Benoist: "La promoción del individuo entraña, pues, un largo proceso de desagregación de lo social que va a conducir a la anomia y a la atomización. El vínculo social, con la modernidad, se hace pura contingencia". Alain DE BENOIST, Más allá de la Derecha y la Izquierda, (antología a cargo de Javier Ruíz Portella), Altera, Barcelona, 2010, p. 164.

14 "Entrevista a Charles Champetier": Hespérides, n. ${ }^{\circ} 16 / 17$, p. 701.

15 "Qué es lo que esconden las peroratas hiperhumanitarias, hiperhigualitaristas, hiperdemocráticas arengadas por los partidarios del Estado-Providencia?...¿¿No se querría construir por casualidad un "homo occidentalis", habitante de una "americanosfera", en la que la diferencia entre el socialismo y el liberalismo no tendría sentido más que durante el circo electoral?". Alain DE BENOIST y Guillaume FAYE, Las ideas de la Nueva Derecha. Una respuesta al colonialismo cultura, Ediciones de Nuevo Arte Thor, Barcelona, 1986, p. 160.

16 "Editorial": Éléments, n. ${ }^{\circ} 56$ (1985).

17 De Benoist y Faye, Las ideas de la Nueva Derecha, p. 180.
} 
del Kali-Yuga, en la terminología sánscrita usada por Julius Evola y por Mircea Eliade (1907-1986), designa una época en la cual la verdad yace sepultada por la ignorancia a la espera de redentores.

Los activistas de la ND europea se ven a sí mismos como unos redentores. Unos visionarios que, siguiendo a Friedrich Nietzsche, creen que los pueblos que tienen una más larga memoria son los que disfrutarán de un futuro más esplendoroso. Se creen visionarios y revolucionarios, ya que el contexto europeo está hegemonizado por materialistas valores burgueses: mercantilismo, individualismo, igualitarismo y universalismo. Todo ello un desastre fruto de una concepción errónea del hombre. Por el contrario, la ND propugna el predominio de la política sobre la economía, de la comunidad sobre el individuo, de la jerarquía sobre la igualdad y de la heterogeneidad sobre la homogeneidad universalista. Como afirma Carl Schmitt, en cita reproducida por Benoist:

[L]a noción esencial de la democracia es el pueblo, y no la humanidad. Si la democracia tiene que seguir siendo una forma política, sólo hay democracias del pueblo y no democracia de la humanidad ${ }^{18}$.

\section{Diagnóstico}

La opinión de la Nueva Derecha europea sobre la realidad occidental en el momento presente no puede ser más negativa. Vivimos una época de total decadencia, adocenamiento y alienación. La ND española a finales del siglo veinte denunciaba una tendencia progresiva e inexorable hacia la muerte. Unos pocos años más tarde, en junio de 2002, insistían proclamando un nuevo Manifiesto contra la muerte del espíritu y de la tierra. Por su parte la ND francesa habla de umbral de esterilidad y de senilidad al referirse a la civilización europeo/occidental. Una profunda pérdida de sentido esteriliza a la sociedad contemporánea. Según su apocalíptica visión:

Como ha visto Konrad Lorenz, la civilización occidental nos arrastra a una muerte lenta. Sus modos de vida tienen incidencias psicológicas, neuróticas y patógenas: nos transformamos en seres domesticados y fragilizados en nuestros comportamientos. La decadencia demográfica, la debilidad de carácter, la degradación genética de los occidentales, son hechos confirmados por los propios médicos ${ }^{19}$.

\footnotetext{
${ }^{18}$ De Benoist, Más allá de la Derecha y la Izquierda, p. 149.

${ }^{19}$ Guillaume FAYE, "Critique du système occidental": Orientations, n. ${ }^{0} 5$ (1984), p. 7.
} 
El hipermaterialismo, el productivismo, el igualitarismo y el hedonismo narcisista ahogan toda trascendencia, espiritualidad y belleza. El culto individualista al bienestar embrutece al hombre, lo convierte en un ser alienado, corrupto y lo incapacita para desarrollar sus posibilidades tanto como miembro de su comunidad como personalmente. Culto individualista que el dualismo cristiano había preparado al plantear que la auténtica vida era la futura vida en el cielo. El liberal, simplemente, sitúa al cielo en la tierra. Y de esta forma cada individuo se afana en lograr el mayor bienestar material posible en una sociedad entendida como una agregación despolitizada de átomos independientes y soberanos.

El corolario político de esta "alienante" realidad es el papel que cumple el Estado, juzgado como un simple y despolitizado regulador del sistema: “...un organismo técnico al servicio de la economía" ${ }^{20}$, cuya auténtica razón de ser sistémica, por tanto, no es la dirección política de la sociedad sino garantizar los derechos individuales para la salvaguarda de la maximización de las inversiones realizadas. Todo ello complementado con los criterios igualitaristas de los Estados de Bienestar y el universalismo de los Derechos Humanos.

Incluso los Estados-Nación, a pesar de su pervivencia, están siendo superados por una tecnoestructura mundial, el auténtico gobierno en la sombra político-económico del planeta Tierra. Siempre según la lectura de la ND europea, este sistema pervive exitosamente por el grado de hegemonía ideológica que ha logrado, dado el gran nivel de interiorización de sus finalidades por parte de sus integrantes. De ahí que sólo exista una leve necesidad de coordinación política reguladora.

La economía se ha subordinado a la política, y los ciudadanos creen ser felices trabajando frenéticamente y consumiendo compulsivamente. En una sociedad, podríamos añadir desde nuestra propia perspectiva, de economía y sociedad de mercado en donde mercancías producen mercancías en un proceso circular constante. Esta sociedad de mercado para la ND es inaceptable - el término que utilizan es el de cancerización - no sólo por sus consecuencias de anomia, alienación y embrutecimiento, sino porque ahoga la auténtica esencia primigenia del hombre: su capacidad de ser comunitaria mediante su voluntad individual. El gobierno de los hombres se ve reemplazado por la administración de las cosas en donde todo se convierte en mercancías: productores, consumidores e incluso necesidades (en ocasiones de una radical falsedad). Como producto final de la combinación siempre de los mismos ingredientes: las estructuras económicas

\footnotetext{
${ }^{20}$ Alain DE BenOIST y Guillaume FAYE, “Contre 1'Etat-providence”: Éléments, n. ${ }^{\circ} 44$ (1983).
} 
supranacionales, la ideología universal e igualitaria de los derechos humanos y la subcultura mundial de masas.

Desaparecido el modelo soviético por su ineficacia económica y autoritarismo, el enemigo principal para la ND pasa a ser el liberalismo como ideología y sistema de valores, y los Estados Unidos - denominada como nueva Cartago - como líder occidental de la modernidad; un país que nace burgués, sin aristocracias de sangre y sin tradiciones ${ }^{21}$. El liberalismo, según sus criterios, es una filosofía política y una ideología totalmente errónea: hace del individuo abstracto la clave de bóveda de todo su sistema. En el terreno político, el liberalismo tiene un consustancial fondo anárquico: el régimen ideal es aquel que establece la menor autoridad posible. Mientras, en un plano social, consuma la ruptura con el principio holista y niega la noción de interés colectivo, siendo la sociedad una mera agregación de individuos que se agrupan para la mejor protección de sus intereses particulares. Por eso, expone la ND francesa:

[E]1 liberalismo es una máquina de producir desilusión...jamás como en el momento presente la anomia social había sido tan grande...el liberalismo destruye las identidades colectivas, las culturas enraizadas y es generador de uniformidad...combatir el liberalismo es combatir el mal de raíz ${ }^{22}$.

Sin embargo, la situación histórica existente, a pesar de su gravedad, permite una esperanza como consecuencia de la propia profundización del problema. Para Faye, en el siglo veintiuno se va a producir una confluencia de catástrofes - económica, social, política, ecológica, migratoria - que hará inviable la reproducción del sistema y obligará a un cambio de civilización. En años anteriores Faye ya había argumentado que, a partir de un cierto límite, la regulación de un sistema en crisis no es posible ${ }^{23}$. Al producirse un colapso civilizatorio, los valores e idearios que lo legitiman perderán su hegemonía y nuestro autor aboga porque las alternativas que surgirán sean revolucionarias: orientadas hacia visiones del mundo orgánicas.

21 "América está entre nosotros: fórmula terrible, que si llegase completamente a ser verdad, querría significar que ya somos muertos vivientes". Guillaume FAYE, Actes du XV colloque national du GRECE, Le Labyrinthe, Paris, 1982, p. 47.

22 "Editorial": Éléments, n. ${ }^{\circ} 68$ (1990).

${ }^{23}$ Guillaume FAYE, "La modernité: Ambiguités d'une notion capitale": Études et Recherches, n. ${ }^{\circ} 1(1983)$, p. 5. 


\section{OBJetivos}

El objetivo genérico de la ND es el despertar de la conciencia de los europeos y este despertar es un combate tan ineluctable como necesario, puesto que, según su propio análisis, se lucha porque no combatir es morir, porque el mundo que nos rodea es el de la pasividad y el sueño, donde la energía del pueblo se muere ${ }^{24}$. Se trata de tomar el relevo de las ideologías dominantes (vistas como ramas de un tronco común) a partir de haber reconstruido una visión del mundo alternativa enraizada con la auténtica identidad europea. La ND afirma haber elaborado un sofisticado nuevo paradigma teórico que supera la obsolescencia de los esquemas tradicionales de derecha e izquierda. Una tercera vía ideológico-política más allá de la izquierda, revolucionaria o moderada, y de la derecha, moderada o extremista. Según sus criterios la auténtica división política del siglo veintiuno no se da entre derecha e izquierda sino entre universalistas e identitaristas. De ahí que al izquierdista lema del nuevo movimiento social "SOS Racismo" oponen su consigna identitaria y diferencialista "SOS Raíces" 25.

La meta final es un mundo heterogéneo constituido por grandes unidades territoriales étnicamente homogéneas, la unión sin confusión según Benoist, y por eso se reivindica, como factor esencial, el derecho a la diferencia y el derecho de los pueblos. Como ya expusiera Joseph de Maistre (1753-1821), la ND opina que no existen los hombres de una humanidad en abstracto sino los individuos que forman diferentes pueblos, etnias y culturas. De ahí que sea imprescindible "pronunciarse por las doctrinas etnonacionales, contra el pacifismo y el humanitarismo"26.

La ND europea tiene unos objetivos estratégicos y tácticos muy claramente definidos, siendo lo más relevantes: potenciar un nuevo-antiguo concepto de libertad comunitaria; sustituir la hegemonía de los valores burgueses por valores aristocráticos; resucitar Europa mediante la reivindicación de su auténtica identidad pagana e indoeuropea; revitalizar la idea de comunidad dotándola de metas y sentido; separar los conceptos jurídicos de nacionalidad y ciudadanía; primar los criterios etnonacionalistas; combatir el igualitarismo ${ }^{27}$ y el universalismo;

\footnotetext{
${ }^{24}$ Guillaume FAYE, "Pour un Gramscisme de Droite": Actes $d u$ XVI colloque national $d u$ GRECE, Le Labyrinthe, Paris, 1982.

${ }^{25}$ Consigna lanzada por Benoist en el XIX Coloquio Nacional de GRECE (noviembre de 1985).

${ }^{26}$ De Benoist y FaYe, Las ideas de la Nueva Derecha, p. 472.

27 "El igualitarismo supone el hundimiento de todo lo que es elevado y diferenciado dentro de lo que es homogéneo, indiferenciado, equivale, de hecho, a la inversión de las jerarquías". "Editorial": Éléments, n. ${ }^{\circ} 28$ (1979)
} 
desmercantilizar el mundo supeditando la economía a la política; lograr la armonía con la naturaleza y una ecología integra ${ }^{28}$ preservando la biodiversidad; y finalmente, conseguir cambiar la democracia representativa por una auténtica democracia participativa y plebiscitaria.

Conseguir todos estos objetivos regeneraría a Europa y le permitiría superar su decadencia y recuperar su genuina esencia, enlazándose pasado, presente y futuro. Para ello las ideas "sanas" de la ND deben cobrar vida en amplios y transversales movimientos políticos regeneradores de las patrias. Como expone Faye:

El futuro pertenece a las revoluciones culturales, espirituales y nacionales. El futuro pasa por la destrucción del orden económico internacional y por la consecución de una idea que ya está en camino: la concentración de espacios económicos autónomos en torno a grandes áreas culturales ${ }^{29}$.

\section{Visión del Mundo, CONCePción del Hombre, de la Naturaleza y de la Historia}

La visión del mundo de la ND está articulada en torno a una serie de convicciones, verdades per se o primeros principios ontológicos y axiológicos. Por ejemplo, la convicción de que en la naturaleza existen unas leyes comunes a todos los seres vivos, extrapolables a los individuos y comunidades humanas: la selección, la desigualdad y la jerarquía son valorados como entes tan naturales como la tierra o el mar. Otra de estas concepciones básicas sería la hipótesis de la importancia de los contenidos informacionales filogenéticos que las comunidades humanas se transmiten de generación en generación y que se van incorporando a lo largo de la historia. Una tercera idea sería que una de las leyes generales de la vida es el conflicto y, por ello, combatir es vivir ${ }^{30}$, siendo la debilidad la antesa-

${ }^{28}$ La valoración de la ecología es de los puntos que la ND francesa ortodoxa ha variado, desde el desprecio inicial a la aceptación de sus críticas radicales al capitalismo desarrollista. Contrastar los primeros editoriales y los de fechas posteriores de Éléments. Ver, por ejemplo: n. ${ }^{\circ} 21 / 22$ (1977) y n. ${ }^{\circ} 79$ (1994).

${ }^{29}$ Guillaume FAYE, "Pour en finir avec la civilisation occidentale": Éléments, n. 34 (1980), p. 9 .

30 "[El hombre posee] un temperamento agonal que hace de la lucha $-\mathrm{y}$ en principio la lucha consigo mismo - la esencia misma de la vida". De Benoist y FAYE, Las ideas de la Nueva Derecha, p. 198. 
la de la muerte ${ }^{31}$. Finalmente, una cuarta sería que la voluntad es un factor esencial para los seres humanos si quieren vivir una existencia auténtica, adecuada a sus potencialidades. Como expone el propio Benoist en famoso artículo publicitado, profusamente, en diferentes publicaciones de las ND europeas: no se trata de buscar una verdad objetiva, exterior al mundo, si no de crear una voluntariamente, a partir de un nuevo sistema de valores. Se trata de fundar un neopaganismo que permita la realización de un modo de existencia auténtico y pleno ${ }^{32}$.

La visión de la ND es empirista: sólo la naturaleza, incluida el hombre, es observable; por lo tanto Dios o no existe o es naturaleza y, por tanto el hombre también es, o puede ser, Dios. Y de la observación como método la ND extrae que la vida humana tiene, o desea tener, un ansia de trascendencia (de ahí que planteen la imbricación de la ontología, la metafísica, la antropología e incluso lo sacro) que el racionalismo ilustrado o post-ilustrado no contempla. Asustada, la Razón - piensan - se niega a tener en cuenta todo lo que no es Logos, y al hacerlo vive en el error. Hace constantemente la guerra pero la rechaza; el derecho es fuerza pero se niega a admitirlo ${ }^{33}$, proclama la igualdad sin conseguirla nunca y rechaza la autoridad que no se base en argumentaciones utilitaristas.

Por todo ello la visión del mundo de la ND es alternativa al "pensamiento único" de la modernidad: el burgués se equivoca en casi en todo menos en el cálculo económico, y la desaparición de los valores aristocráticos ha representado para Europa una catástrofe, sólo comparable a la sustitución del paganismo en las comunidades indoeuropeas por el dualista ${ }^{34}$, universalista e igualitario cristianismo. Por tanto la realidad, según la observa la ND, es esquizofrénica: por un lado, el capitalismo demuestra la supervivencia de los más fuertes y aptos en un combate de todos contra todos, pero, a la vez, el liberalismo pretende y ha consegui-

31 "La etología y la biología moderna han demostrado...la importancia de los comportamientos de caza y guerra en la formación filogenética de la humanidad, como criterios de base de la selección natural y de la orientación de la evolución. Somos los “hijos de Caín”". Ibid., p. 299.

${ }^{32}$ Alain DE Benoist, "La religion de l'Europe": Éléments, n. ${ }^{\circ} 36$ (1980), p. 20.

33 "La existencia de los hombres demuestra, por otra parte, que éstos, a pesar de sus protestas, han asociado siempre la fuerza y el derecho...Bismarck..."la fuerza precede al derecho". Alain DE BENOIST, "Ni fraiche ni joyeuse": Éléments, n. ${ }^{\circ} 24 / 25$ (1982), p. 28.

34 "Cuando se trata de especificar los valores propios del paganismo, se ha enumerado rasgos tales como una concepción eminentemente aristocrática de la persona humana, una ética fundada sobre el honor (la "vergüenza" más que el "pecado"), una actitud heroica ante los desafíos de la existencia, la exaltación y la sacralización del mundo, de la belleza, del cuerpo, de la fuerza y la salud, el rechazo del "otro mundo", inseparabilidad de la estética y la moral, etc. Todo esto nos parece exacto, pero en cierta medida secundario. El rasgo fundamental, en nuestra opinión, es el rechazo del dualismo". De Benoist y FAYE, Las ideas de la Nueva Derecha, p. 191. 
do domesticar al hombre occidental mediante el narcótico de los Derechos Humanos, ideología absurda de la modernidad en la medida que parte de la convicción de que todos los seres humanos son iguales y libres. Y, por el contrario, la ciencia (etología, biología, antropología) "demuestra" que los seres humanos son profundamente desiguales.

Y tampoco libres. La ND rechaza taxativamente una visión de la libertad individual, consecuencia de la creencia de que los seres humanos son titulares de unos derechos inalienables inherentes a la persona y, por ello, no concedidos por ninguna instancia superior. La concepción de la libertad de la ND, muy bien explicitada por Evola - siguiendo a Nietzsche-, propugna que no hay una libertad general y abstracta sino libertades articuladas según la naturaleza propia de los seres. La libertad pertenece a un plano práctico y político y no al filosófico o moral. Debe ser conquistada: no existen beneficiarios espontáneos, sino únicamente fundadores y garantes. Nadie nace libre, pero algunos lo llegan a ser. La libertad resulta de la acción hecha para instaurarla o para apoderarse de ella. Tal acción puede ser el resultado de los individuos o de las colectividades ${ }^{35}$.

Se es libre, por tanto, como individuo, por pertenecer a una comunidad. Y, como el todo es superior a la suma de las partes y posee cualidades que le son propias (holismo), el Estado debe primar no el bienestar material — prosaico objetivo - de los ciudadanos sino la garantía de su existencia y de su poder en la historia. De ahí que la felicidad también deje de ser un tema individual para pasar a una concepción colectiva, puesto que —otra creencia básica de la ND— los protagonistas de la Historia son los pueblos, las comunidades, las naciones y/o las culturas en una constante dialéctica de confrontación. Para la ND el hombre no tiene naturaleza sino cultura e historia, que se desarrollan a partir de unas características biológicas constantes: siempre — afirman — habrá cazadores y guerreros entre nosotros, fuertes y débiles, hombres superiores y hombres inferiores ${ }^{36}$, aunque los partidarios de los Derechos Humanos no quieran reconocerlo y se nieguen a admitirlo. La visión del mundo, por tanto, de la ND es organicista, pluralista y diferencialista.

35 "Esta concepción de la libertad humana está estrechamente unida a una cierta concepción de la historia: la "naturaleza", lo innato, el pasado, condicionan el futuro del hombre, pero no lo determinan. Es en este espacio semántico entre "condicionar" y "determinar" en donde yace la libertad, el hombre no puede obrar más que con lo que tiene, pero con lo que tiene, puede ser y hacer lo que quiera". De BENOIST, "La religion de l'Europe", p. 12.

36 "[N]ada autoriza a decir que la razón está igualmente repartida en todos los Hombres". DE Benoist y Faye, Las ideas de la Nueva Derecha, p. 397. 
Por eso la concepción jurídica de los derechos individuales de la ND plantea una opción no universalista. Propone que cada comunidad política, histórica y cultural debe tener reconocido su derecho a establecer, como crea oportuno, cuáles son los derechos de sus miembros (de ahí su criterio de distinguir entre ciudadanía y nacionalidad). Y, obviamente, los derechos los concede la comunidad al individuo. El razonamiento consiguiente plantea que, si los hombres no pueden ser valorados igual, tienen diferentes necesidades y pertenecen a diferentes comunidades humanas, ¿por qué establecemos unos mismos derechos universales y abstractos para todos cual lecho de Procusto?

Sólo se explica por el triunfo en el mundo de unos valores burgueses y cristianoides: individualistas, economicistas, igualitaristas y universalistas, basados en una errónea visión del mundo y del hombre, siendo la misión de la ND, mediante el combate ideológico cultural y metapolítico, advertir al mundo de sus errores y propagar otra visión del mundo alternativa, visionaria hacia el futuro y lúcida respecto al presente y al pasado.

Tan lúcida y potente es esta visión y el ideario que la explicita que, en su opinión, es capaz de armonizar pares en teoría antagónicos (como en su día pretendiera el Fascismo Clásico) ${ }^{37}$ : instinto y cultura; racionalidad e irracionalidad; hipermodernismo y postmodernismo y tradición; ecologismo y desarrollo tecnológico; nación y comunidad supranacional (Europa); soberanía máxima del Estado y libertad económica individual; derecha e izquierda; sacrificio individual y libertad y/o felicidad colectiva. Immanuel Kant (1724-1804) y Karl Marx (18181883) se equivocan, mientras Nietzsche, Heidegger y Evola aciertan. La sangre vale más que el oro ${ }^{38}$. El hombre, como el resto de los seres vivos, es un ser nacido para combatir, asentado en un territorio y desigual, además de tender a la trascendencia y poder forjar (algunos hombres y algunos individuos) su destino. Más allá del bien y del mal cristiano, liberal o socialista. El modo de estar en el mundo fundamenta el derecho a transformarlo.

En lógica coherencia con todos los elementos anteriormente expuestos cabe destacar que la concepción de la libertad de la ND (coordinada con su visión social organicista) es, como lo plantea Nietzsche, ilimitada. Los hombres libres son sujetos y objetos de su existencia y, por ello, el mundo les pertenece. Manifestar su libertad supone explicitar su autonomía. Y como expresa Benoist:

${ }^{37}$ ANTÓN-MELLÓN, "Las concepciones nucleares, axiomas e ideas-fuerza del Fascismo Clásico (1919-1945)".

38 "Desde las piedras levantadas en Stonehenge a los símbolos táuricos de Cnossos, se ha expresado siempre la misma idea: la sangre vale más que el oro. He aquí la pesadilla de los auténticos fundadores de Occidente”. De Benoist y FaYe, Las ideas de la Nueva Derecha, p. 474. 
[S]ólo puede ser libre el hombre que se expresa creando. Ahora bien, no hay creación "pura". Se crea siempre a expensas de un objeto - ya sea la "naturaleza" u "otro-hombre-tomado-como-naturaleza"-...se apropia, no solamente como útiles y como instrumentos, sino también como "prolongaciones" y "partes" de su cuerpo. De los objetos (materiales y humanos) sobre los que se ejerce su acción ${ }^{39}$.

Como es obvio, los planteamientos económicos de la ND (además de parte de los políticos) se derivan de estos criterios base o concepciones nucleares. El capitalismo es un sistema productivo idóneo siempre que esté controlado por poderes políticos etno-nacionales. En líneas generales la visión del mundo de la ND es la antítesis de la concepción burguesa-liberal del mundo y de sus valores. Se reivindica lo sacro, lo irracional y el espíritu de aventura, como complemento necesario a una racionalidad técnica imprescindible para realizar lo que se cree propio de los seres humanos: su voluntad de poder para forjarse destinos colectivos. A la contra del proceso histórico que conocemos como modernidad, la ND propugna que los seres humanos europeos, si quieren desarrollar sus potencialidades, deben eliminar la alienante domesticación cristiano-liberal a la que han sido sometidos. De esta forma la generosidad substituiría al cálculo, el idealismo/altruismo al materialismo/pragmatismo, el sacrificio al hedonismo, y la aventura colectiva a la comodidad egotista. El enriquecedor combate, piensan, factor de superación constante, condicionaría el ser de hombres y comunidades. Para la ND europea, sin fisuras ni políticas ni ideológicas ${ }^{40}$, una adecuada forma de "estar en el mundo" tomaría el lugar de una absurda manera de vivir, tan alienante como sin sentido y norte.

El análisis realizado hasta el momento presente permite plantear que el ideario de la ND se mantiene en los esquemas prototípicos de la Derecha al levantar la banderas de la desigualdad, la visión de la vida como combate, la tradición y la concepción orgánica de las comunidades étnicamente homogéneas y puede ser juzgado como un posible canto del cisne de los valores aristocratizantes de

${ }^{39}$ Alain DE BENOIST, "Nature et culture", en Pierre VIAL, Pour une renaissance culturelle. Le GRECE prend la parole, Copernic, Paris, 1979, p. 92.

${ }^{40}$ A lo largo de la trayectoria política de la ND francesa las escisiones se produjeron en sus inicios, años setenta del pasado siglo, cuando un grupo de miembros de GRECE se separaron de la ND y fundaron el Club de L'Horloge. Siendo el factor de desunión de estos la admisión de los parámetros básicos del liberalismo. Por otra parte otro grupo de miembros de GRECE (J. C. Barder, Vial o J. Y. Le Gallou), en los años ochenta, decidió apoyar activamente a Jean Marie Le Pen y al FN. En 1986 Faye decidió abandonar GRECE. 
las sociedades europeas de Antiguo Régimen enfrentadas a la Modernidad. Lo aristocrático como contrapunto a lo burgués ${ }^{41}$. Sería un survival en la terminología de la Antropología ${ }^{42}$. El propio Alain de Benoist nos confirma esta hipótesis al revelarnos, en el "Prefacio" de su obra, Le grain de sable, que el origen del seudónimo que más ha utilizado, Robert de Herte, es un homenaje a un pariente materno, Charles-Germain de Herte, aristócrata y teniente de regimiento de mosqueteros que fue guillotinado durante la Revolución francesa a los 38 años de edad.

\section{Concepciones nuCleares de la ND y del FASCismo Clásico (1919-1945). UNA IDENTIDAD NO IDÉNTICA}

Establecidas las ideas-fuerza de la ND europea, constatemos cuáles son sus parámetros cultural-políticos de referencia, a quienes consideran los grandes clásicos de la historia de las ideas. Según el objetivo inicial que planteamos de determinar su posible adscripción al campo de la Derecha Radical/Extrema Derecha y el grado de continuidad con el Fascismo Clásico.

La ND francesa ${ }^{43}$ en 1996, en una sección didáctica de su órgano de expresión oficial Éléments, recomienda leer a los dieciséis autores y obras que a continuación se exponen:

- Friedrich Nietzsche: La volonté de puissance.

- Georges Sorel: Réflexions sur la violence.

- José Ortega y Gasset: La révolte des masses.

- Maurice Barrès: Scènes et doctrines du nationalisme.

- Maurice Barrès: Le voyage de Sparte.

- Charles Maurras: L'avenir de l'intelligence.

- Joseph de Maistre: Considérations sur la France.

- Abel Bonnard: Les modérés.

- Armin Mohler: La révolution conservatrice en Allemagne, 19181932.

41 “'[L]os 'nuevos burgueses'...son solamente quienes, en un mundo enteramente modelado por la mentalidad burguesa, son una caricatura de los antiguos modos aristocráticos...el gusto por lo inútil, lo gratuito, el sentido del gesto, el honor, el don, es decir todo lo que a sentido a la existencia”. Editorial de Éléments, n. ${ }^{\circ} 72$ (1991).

${ }^{42}$ Las positivas connotaciones del calificativo "noble" sería un ejemplo.

${ }^{43}$ Éléments, n. ${ }^{\circ} 86$ (1996). 
- Arthur Moeller van den Bruck: La révolution des peuples jeunes.

- Silvio Vietta: Heidegger, critique du national-socialisme et de la technique.

- Thierry Maulnier: Au-delà du nationalisme.

- Werner Sombart: Le socialisme allemand.

- Carl Schmitt: Du politique. "Légalité et légitimité" et autres essais.

- Ernst Niekisch: Hitler une fatalité allemande et autres écrits nationaux-bolcheviks.

De forma paradigmática esta selección, obras publicadas por Labyrinthe (editorial oficial de GRECE) o en colecciones dirigidas por miembros de GRECE, se inicia con una obra de Nietzsche y otra sobre Heidegger ${ }^{44}$. En ella se recogen textos clásicos del ultranacionalismo francés (Barrès, Maurras); las obras capitales sobre y de la Revolución Conservadora Alemana; además de incluir a clásicos como Ortega y Gasset y de Maistre, así como la curiosidad de revitalizar a un olvidado, el líder intelectual de los nacional-bolcheviques alemanes, Niekisch.

De todos ellos merece la pena destacar el grupo de autores de la Revolución Conservadora Alemana (Mohler, Moeller van den Bruck, Sombart y Schmitt) dada la importancia de la influencia que han ejercido sobre la ND. De tal modo que sus ideólogos más destacados se identifican con ese movimiento cultural del primer tercio del siglo pasado y se ven a sí mismos como los revolucionarios conservadores de los siglos veinte y veintiuno ${ }^{45}$. El propio Faye así lo explicita:

La actitud que nosotros tomamos frente a la modernidad se puede entender como la prolongación — pero evidentemente no el calco — de las posiciones de los principales pensadores de la "Revolución Conservadora" $"$.

${ }^{44}$ La gran influencia de Nietzsche y Heidegger sobra la ND podría ser objeto de otro artículo.

${ }^{45}$ En 1993 Alexander Douguin, líder de la ND rusa, escribe en la revista Vouloir de la ND belga que para alcanzar la paz euroasiática se tiene que lograr ser unos sujetos nacionales libres y tradicionales, guiados por los principios de propias revoluciones conservadoras. Alexander DouGUIN, "Crise balkanique, crise européenne": Vouloir, n. ${ }^{\circ}$ 97/100 (1993), p. 37.

${ }^{46}$ Guillaume FAYE, "La modernité: Ambiguités d'une notion capitale": Etudes et Recherches, n. ${ }^{\circ} 1(1983)$. 
Los revolucionarios conservadores alemanes son un referente para la ND, colectiva e individualmente. Varios ejemplos. De los 248 artículos publicados en la revista de la ND italiana Trasgressioni entre 1986 y 2002, de los artículos de autores considerados como clásicos, veinte han sido textos de Ernst Jünger (1895-1998) (9), Schmitt (6), Oswald Spengler (1860-1936) (3), Sombart (1) y Knut Hamsun (1859-1942) (1). En el número 19 de la revista de la ND española Hespérides, se anuncia la próxima aparición de un número monográfico sobre la Revolución Conservadora alemana, número que no ha llegado a publicarse por la desaparición de la revista. Esa admiración se convierte en devoción por lo que se refiere a la figura de Jünger. El primer libro publicado por la ND española en su colección Los Libros de Hespérides ha sido el de Alain de Benoist: Ernst Jünger y el trabajador. La ND francesa ha editado numerosos textos de y sobre este autor, por ejemplo Dominique Venner publicó en Éléments en 1995 un artículo titulado "Jünger: la figure même de 1'Européen". El propio Benoist, a partir de 1989, dirigió la colección "Revolución Conservadora" de la Editorial Pardés (fundada por discípulos franceses de Evola), y en 1998 publicó una obra titulada Ernst Jünger: une bio-bibliographie, en la editorial de GRECE Le Labyrinthe.

En 1932 Edgar J. Jung (1894-1934), secretario del líder político conservador Franz von Papen (1879-1969), escribe en su obra Deutschland und die Konservative Revolution:

Llamamos Revolución Conservadora a la recomposición de todas aquellas leyes y acciones elementales en ausencia de las cuales el hombre pierde el contacto con la naturaleza y con Dios, viéndose incapacitado para edificar un orden verdadero. En lugar de la igualdad proponemos los valores interiores, en lugar de la orientación social la apropiada integración en una sociedad jerárquica, en lugar de la elección mecánica el surgimiento orgánico de jefes auténticos, en lugar de la coerción burocrática la responsabilidad personal de una auténtica autodisciplina, en lugar de la felicidad de las masas el derecho de la comunidad del pueblo ${ }^{47}$.

Según Keith Bullivant, los criterios fundamentales de los revolucionarios conservadores alemanes fueron: cuestionarse la primacía de la racionalidad; el rechazo a la militancia partidista; pretender sustituir la democracia por un sistema autoritario y jerárquico; rechazar lo que denominaban "viejo conservaduris-

${ }^{47}$ Edgar Jung, citado por: Keith Bullivant, "La Revolución Conservadora”, en Anthony Phelan (ed.), El dilema de Weimar. Los intelectuales en la República de Weimar (1985), Edicions Alfons el Magnànim-Institució Valenciana d'Estudis i Investigació, Valencia, 1990, p. 88. 
mo"; valorar positivamente las experiencias guerreras y, por último, potenciar eternos valores vitalistas para superar la decadencia ${ }^{48}$. De esta forma el rechazo a la decadencia y una firme voluntad de defender una concepción del mundo jerárquica, orgánica, comunitarista y guerrera constituyen la razón de ser o alma mater de la Revolución Conservadora alemana. Estas concepciones nucleares son compartidas por la ND europea. De ahí que en los años iniciales de GRECE, en 1977, en un editorial de Éléments (n. ${ }^{\circ} 20$, febrero/abril) titulado "La Revolución Conservadora", se exponga lo interesante de los análisis y criterios de estos autores como colectivo y se abogue por una nueva revolución conservadora.

Esta concomitancia de criterios y objetivos entre antiguos y nuevos conservadores revolucionarios es uno de los argumentos de mayor peso que utiliza el profesor británico Roger Griffin para explicitar el carácter esencialmente fascista de la ND europea ${ }^{49}$, según el tipo ideal weberiano que establece. A partir del cual el fascismo (genérico) debe verse como una forma de ultranacionalismo revolucionario, cuyo mito movilizador es la visión del renacimiento de la nación en un nuevo orden post-liberal que ponga fin al período de agudo declive y decadencia. Y por ello el argumento central que utiliza es el del común diagnóstico realizado por la ND y el Fascismo Clásico de la existencia de una crisis y decadencia civilizatoria que deben ser superados por amplios y transversales movimientos políticos regeneradores de las patrias en procesos palingenésicos. Este aspecto central de la decadencia también es subrayado por otra autoridad académica en el estudio del Fascismo Clásico, Robert O. Paxton, al afirmar en el inicio de la definición que efectúa que "se puede definir el fascismo como una forma de conducta política caracterizada por una preocupación obsesiva por la decadencia de la comunidad" 50 .

El carácter palingenésico-nativista ${ }^{51}$ de las propuestas políticas de la ND y del Fascismo Clásico en sus aspectos ideológicos nos conduce a la necesidad de subrayar otro de los aspectos centrales de ambos idearios: la importancia clave de la idea de comunidad según planteamientos ultranacionalistas ${ }^{52}$. Se trataría de un auténtico fetichismo de la identidad colectiva en la terminología de Bihr.

${ }^{48}$ Bullivant, "La Revolución Conservadora".

${ }^{49}$ GRIFFIn, "Plus ça change! El pedigrí fascista de la Nueva Derecha".

${ }^{50}$ Robert O. PaXTon, Anatomía del Fascismo, Crítica, Barcelona, 2005.

${ }^{51}$ Sobre el concepto nativismo ver: Cas Mudde, Populist Radical Right Parties, Cambridge University Press, New York, 2007; y Hans-George BETz, "Contra el 'totalitarismo verde': nativismo antiislámico en los populismos radicales de derecha en Europa occidental”, en SIMÓN (ed.), La Extrema Derecha en Europa desde 1945 a nuestros días.

${ }^{52}$ Mudde, Populist Radical Right Parties. 
Patrias con un destino colectivo a desarrollar, y por ello necesariamente homogéneas; racial o culturalmente puras en la propuesta fascista, o respetando las diferencias étnicas, evitando el universalismo y el mestizaje multiculturalista, en las propuestas de la ND y en lo que ha venido en llamarse racismo diferencialista o culturalista ${ }^{53}$.

Por otra parte, la reivindicación de las desigualdades humanas como un factor natural, enriquecedor y socialmente útil también es un relevante factor compartido entre la $\mathrm{ND}^{54}$ y cualquier discurso que puede ser considerada adscrito a la gran familia ideológica de la derecha. La cual incluye diferentes subfamilias o subconjuntos como todos aquellos que pueden ser agrupados en el término Derecha Radical. A su vez, esta está formada por otros subconjuntos entre los que merece destacarse, por su gran coherencia ideológica y política, el fascismo. Las analogías entre la ND y el fascismo clásico vienen dadas por factores, previamente expuestos, como compartir un mismo o parecido diagnóstico y una misma o parecida visión del mundo a partir de los referentes filosófico-culturales utilizados por ambos; todo ello a partir de una idéntica concepción del hombre, la naturaleza y la historia: el hombre visto como un ser agresivo, jerarquizado y territorializado ${ }^{55}$. Los descendientes de Caín, según afirma el propio Benoist. Siendo el factor de la agresividad y la exaltación de la lucha un factor decisivo de la vida humana otro elemento de concomitancia entre la ND y el fascismo, entendido el eterno combate de un modo socialdarwinista extremo en la opción fascista, y de una forma metafísica-existencial en la ND. De ahí que la ND rechace el socialdarwinismo, e incluso al fascismo, al que acusa de totalitario y de jacobinismo pardo. El fascismo fracasó y por ello la ND lo analiza como cosa de un pasado caduco. Lo importante son las concepciones nucleares a otro nivel

${ }^{53}$ Xavier ToRrens, "Racismo y antisemitismo", en Joan Antón-Mellón (ed.), Ideologías y Movimientos Políticos Contemporáneos, Tecnos, Madrid, 2006.

54 "GRECE retained an ideological core... This was the defence of identity (of whatever kind) and a refusal of egalitarianism... Ideological contradictions between ND factions did occasionally emerge, but the core ideology remained untouched". [GRECE conservó un núcleo ideológico... La defensa de la identidad (cualquiera que fuera) y el rechazo del igualitarismo... Las contradicciones ideológicas entre las facciones de la ND emergieron ocasionalmente, pero la ideología central se mantuvo intacta]. Tom McCulloch, "The Nouvelle Droite in the 1980s and 1990s: Ideology and Entrism, the Relationship with the Front National": French Politics, n. ${ }^{\circ} 4$ (2006), p. 161.

55 "Social inequality forms the basis of the political axiom of the extreme right. Inequality is perceived in two ways: as social and moral differentiation and as social and moral hierarchy". [La desigualdad social configura las bases del axioma político de la extrema derecha. La desigualdad percibida de dos maneras: como una diferenciación moral y social y como una jerarquía moral y social]. Meindert FenNemA, "Populist Parties of the Right": ASSR Working paper, 04/01 (2004), p. 7. 
superior, el nivel de la Derecha Radical. Sin embargo, la esencia del fascismo se encuentra en la convicción de que la civilización avanza cuando existe un orden político "natural" por el cual los no selectos están al servicio (incluso esclavizados) de los selectos. Y ese es el futuro ordenado que plantea Faye (en continuidad clara entre el fascismo clásico y la ND) como posible, deseable y capaz alternativa de solucionar todos los problemas:

Reflexionar sobre una organización mundial a dos velocidades, del hecho de la imposibilidad tecnosociológica de extender a todo el planeta la lógica del "progreso-desarrollo"...Es posible imaginar y predecir el retorno de una gran parte de la humanidad a las sociedades tradicionales, poco consumidoras de energía, socialmente más estables y más felices, mientras que en el cuadro de una sociedad planetaria, una minoría podría continuar siguiendo el modelo de vida tecnoindustrial. Mañana dos esferas: una nueva Edad Media y la Hiperciencia....arqueofuturismo, asociación explotadora de dos contrarios ${ }^{56}$.

La derrota del fascismo en 1945 supuso su demonización, la marginación política y cultural de sus idearios y la hegemonía de los valores democráticos anti-fascistas en los países demoliberales, cuya explicitación más avanzada fue la constitucionalización de los derechos colectivos en los países que se han definido como Estados sociales y democráticos de Derecho. Ante esta realidad, la supervivencia de los idearios fascistas se efectuó en círculos muy marginales de creyentes, entre otros factores porque el fracaso de la fórmula política fascismo disgregó a las diferentes subfamilias de la Derecha Radical que habían apoyado el desarrollo de los movimientos fascistas ${ }^{57}$ - así como otros modelos autoritarios como el franquismo o el salazarismo - en lo que ha sido denominado "Compromiso Autoritario" 58 , alianza contrarrevolucionaria o "Contrarrevolución Preventiva" ${ }^{59}$. El fascismo se convirtió en una ideología maldita, criminalizada

${ }^{56}$ Guillaume FAYE: "El balance de la Nueva Derecha": UrKultur. Revista Digital Europea Transnacional, n. $^{\circ}$ 5, http://urkultur-imperium-europa.blogspot.com/ (19-09-2011).

${ }^{57} \mathrm{El}$ hecho de que el unitarismo sea la idea-fuerza clave del fascismo no debe hacernos olvidar que los movimientos fascistas allí donde lograron acceder al poder lo hicieron gracias a una amplia coalición de fuerzas de Derecha Radical. Ver: Ferran GALLEGO, Todos los hombres del Führer, Debate, Barcelona, 2006; y Paolo Buchignani, La Rivoluzioni in camicia nera, Mondadori, Milano, 2006.

${ }^{58}$ Philippe Burrin, "Politique et société: les structures du pouvoir dans l'Italie fasciste et l'Allemagne nazi": Annales, n. ${ }^{\circ} 3$ (1998).

${ }^{59}$ Norberto BobBio, "Riforme e rivoluzioni", en Paolo FARneti (ed.), Politica e Società, La Nuova Italia, Firenze, 1972. 
jurídicamente salvo por unas escasas minorías de creyentes y/o simpatizantes obviamente los oportunistas desaparecieron- Esta situación de alejamiento máximo del poder hizo que cobrara una relevancia superior la conservación de las ideas-fuerza que homogenizaron a la Derecha Radical en el primer tercio del siglo veinte. Esta ha sido la muy complicada labor que la ND se impuso reconvertir dichas ideas-fuerza adaptándolas a una época de predominio indiscutido de las ideas y valores democráticos. Una adaptación necesariamente sofisticada ${ }^{60}$ ya que, para que perviva lo estratégico, era necesario desprenderse de lo táctico. Una identidad no idéntica disfrazada de nuevo paradigma más allá de la derecha y de la izquierda ${ }^{61}$.

El fascismo clásico constituyó una fórmula política exitosa en Italia y Alemania de 1922 a 1945, una alternativa de derecha radical/extrema derecha a las miserias, contradicciones y problemas de la modernidad liberal ${ }^{62}$. De la misma forma, la ND ha desarrollado un ideario alternativo a las disfunciones de la posmodernidad desde una óptica de Derecha Radical en los terrenos político y cultural, respetando (igual que en los años treinta del pasado siglo) las jerarquías sociales y el sistema productivo capitalista. Una alternativa que, como la Derecha Radical ha evidenciado en múltiples ocasiones, es accidentalista, lo que ayuda también a explicar las diferencias tácticas, e incluso algunas estratégicas, entre el fascismo clásico y la ND. Por eso la ND puede rechazar el totalitarismo y la violencia como método sistemático de actuación política, y a la vez abogar por una "auténtica" democracia pebliscitaria-representativa y federalista.

Pero todas estas cuestiones no son lo más relevante. Lo relevante es la convicción de la ND de que los protagonistas de la historia son las comunidades étni-

${ }^{60}$ Ver: Alberto SpEKTOROwSKI, "Ethnoregionalism: The Intellectual New Right and the Lega Nord": The Global Review of Ethnopolitics, vol. 2, n. ${ }^{\circ} 3$ (2003).

61 "[T] he fascist matrix...At the heart of fascist thinking was the creation of a new elite of men, who would forge a holistic nation and build a new third way State". [[L]a matriz fascista...La creación de una nueva élite de hombres estaba en el corazón del pensamiento fascista, hombres que forjarían una nación holística y que construirían una tercera forma nueva de Estado]. Roger EATWELL, "The Nature of Fascism: or Essentialism by Another name?": Ethik (2004), p. 2.

62 "[A]n alternative modernity", en: Roger GRIFFIn, Fascism, Totalitarism and Political Religion, Routledge, London/New York, 2005, p. 9; “[E]1 lado oscuro de la modernidad”, en: Michael MANN, Fascistas, PUV, Valencia, 2006, p. 387; "una modernidad resacralizada", en: Emilio GENTILE, "El Fascismo italiano", en Joan Antón-Mellón (coord.), Orden, jerarquía y comunidad. Fascismos, Dictaduras y Postfascismos en la Europa Contemporánea, Tecnos, Madrid, 2002; o "reaccionaria", en: Jeffrey HeRF, El modernismo reaccionario, FCE, México, 1990. 
camente homogéneas ${ }^{63}$; que la libertad sólo está al alcance de unos pocos seres humanos selectos; y que una sociedad humana armónica es aquella en la cual los hombres se definen y actúan como agresivos, jerárquicos y territorializados, donde desarrollan una teoría política e instituciones en consonancia. Todo lo contrario de las concepciones nucleares ilustradas según las cuales los seres humanos nacemos libres, iguales y racionales. Como afirma la expresión latina Tertia non datur (no hay tercera vía) entre la democracia y la barbarie, tanto en el terreno de las ideas, los actos o las instituciones. Tal vez podríamos recordar como conclusión final aquellas sabias estrofas de un viejo tango porteño: "no hay nostalgia peor que añorar lo que nunca jamás sucedió”.

${ }^{63}$ Siendo esta imprescindible homogeneidad un eco, metabolizado, de la idea-fuerza más relevante del fascismo: el unitarismo. Esta es la Idea del fascismo: el unitarismo ultranacionalista palingenésico y violento. ANTÓN-MELLÓN, "Las concepciones nucleares, axiomas e idea-fuerza del Fascismo Clásico (1919-1945)”, p. 61. 